\title{
Tunable fiber laser using concatenated non-adiabatic single-mode fiber tapers
}

\author{
M A Quintela, A Quintela, N. Becue, J M Lázaro, F. Anabitarte and J M Lopez-Higuera \\ Photonic Engineering Group-University of Cantabria, Avda. Los Castros s/n Santander, Spain \\ E-mail: quintelm@ unican.es
}

\begin{abstract}
An erbium doped fiber ring laser (EDFRL) that incorporates four non-adiabatic concatenated single-mode fiber tapers (acting as tunable filter in the laser cavity) is presented. These concatenated fiber tapers integrates a filter with a narrower band-pass and a higher modulation depth than a single taper. The tuning of this filter was implemented applying a controlled perturbation in the fiber taper. The proposed laser architecture was successfully demonstrated in the laboratory in which a tuning range of $20.8 \mathrm{~nm}(1544.5 \mathrm{~nm}-1565.3 \mathrm{~nm})$ were measured.
\end{abstract}

\section{INTRODUCTION}

From more than 20 years the fiber tapers have been used typically as fiber couplers [1] and as optical sensors for physical parameters [2] [3]. One of the most important characteristic of the fiber taper is that the interaction of the light with the environment takes place in its thin zone. This property permits the development of refractive index or humidity sensors [4]. Moreover in the non-adiabatic fiber tapers, it is obtained interferences between the modes (principally the $\mathrm{LP}_{01}$ and the $\mathrm{LP}_{02}$ ), which can be uses, for example, to detect strain and angular movement [5]. The fiber tapers can also be used as tunable optical filters that, in addition to a wide tuning range, the device offers simplicity, cost-effective and reliable fabrication procedure. By introduction of these filters based on tapers inside the resonator cavity, tunable fiber lasers can be obtained. To use tapers in these proposed applications, carefully designs and fabrications must be realized. In this paper, an erbium doped fiber ring laser (EDFRL) using non-adiabatic concatenated single-mode fiber tapers is presented. In order to achieve tunable filters, external perturbations were applied to the fiber tapers.

The paper is organized as follows: in the first part the results of fabricated non-adiabatic single-mode fiber tapers together with their experimental characterization when perturbations are applied are shown; in the second one the experimental results of the constructed EDFRL using these fiber tapers are presented. Finally, conclusions are given.

\section{NON-ADIABATIC FIBER TAPERS FABRICATION AND CHARACTERIZATION}

It known that the adiabatic fiber tapers minimizes the lost from a soft and large transition between the initial diameter and the final diameter of the taper [6]. The non-adiabatic type presents interesting properties just us the capacity of measure external parameters from the light interaction with them in the thin zone. These fiber tapers can be obtained realizing a quick drop of the fiber diameter. The normalized frequency (V) of the fiber core is reduced proportionally with the reduction of the fiber diameter during the taper manufacture. When $\mathrm{V}_{\text {core }}<1$, the fundamental mode $\mathrm{LP}_{01}$ is guided for the cladding and has the possibility to couple with any $\mathrm{LP}_{0 \mathrm{~m}}$ mode, although the principal interaction is with the $\mathrm{LP}_{02}$ mode. When $\mathrm{V}_{\text {core }}$ returns $>1$, the $\mathrm{LP}_{01}$ travels in the fiber core and it is guided in the same form that before to enter in the taper. As conclusion, in a non-adiabatic taper the interaction of the optical mode with the external medium is very high.

The transmitted power in a taper depends on the phase difference between the coupled modes of the taper. In turn, this phase difference depends on the interaction length between the modes and the transmitted wavelength. This response is a quasi-sinusoidal function of the wavelength. This is due to the interference between the two principal modes $\left(\mathrm{LP}_{01}\right.$ and $\mathrm{LP}_{02}$ ). This spectral response can be tuned by changing the phase difference by some means, for example, stretching the taper or changing the external conditions of the taper's surrounding environment.

The fiber tapers used in this work have been made using a custom machine developed in the Photonic Engineering Group at the University of Cantabria (it is observed in http://www.teisa.unican.es/gif/). This computer controlled machine is based on several translations stages and an oxi-butane torch. While two translation stage stretch fiber the torch sweep the fiber, so the taper is made. With the machine it is possible to make both adiabatic a non-adiabatic tapers. 


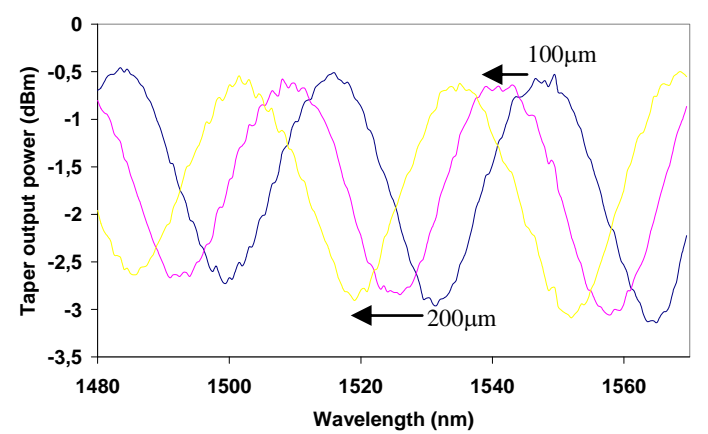

Fig. 1. Spectral response of a non-adiabatic fiber taper and its shifts upon bending.

In this work, in order to obtain a tuning of the tapers, these one were localized between two translation stages. When these translation stages moves in different direction in the axial direction the taper structure is deformed. As illustration of the tunability of these structures a sample of the experimental transmission spectral response of a fiber taper for several rapprochements of the taper ends is showed in Fig. 1.

As was expected, the spectral response is quasi-sinusoidal. Moreover, it shifts towards inferior wavelengths when the taper is deformed. The effect of taper geometry variation in the spectral responses when it is subjected to these perturbations is not still studied. However we are experimentally checked the reproducibility of the fiber taper and their associated effects. The output power of the taper during the displacement ( 0 to $5 \mathrm{~mm}$ ) to a constant speed and the return to the position 0mm for two different wavelengths can be observed in Fig. 2. The return is clearly a mirror of the forward movement proving the reproducibility of geometric configuration of the fiber taper for a specific position of the translation stage.

On the other hand, the ring fiber laser achieves easily lasing even with low concentration erbium doped fibers (EDF) using long cavity lengths (typically $>10 \mathrm{~m}$ ). This implies that these lasers can present multiple longitudinal modes which produce undesired effects such as mode-hopping and large linewidth. In order to achieve a ring fiber laser with a low linewidth, a narrow optical filter is required. The cascading of fiber tapers is a simple method for obtain optical filters with a narrow band-pass [7]. The spectral response of the concatenated tapers is equal to the product of the spectral responses of all of the tapers. As a consequence an ultra-narrow optical filter can be obtained with a multiple tapers in series. Nevertheless, as each taper has a minimum transmission lost of $0.5 \mathrm{~dB}$, the number of fiber tapers in series has to be limited in an agreement between the band-pass and the loss of the filter.

In our experimental demonstration four concatenated fiber tapers were used. Fig. 3 shows the theoretical response of four filters in series which spectral responses have periods of 80, 40, 20 and 10nm. The total spectral response of concatenated tapers is narrower and the modulation depth is higher than a single taper. This permits a better stability of fiber laser and an easier discrimination the lasing wavelength in the unflattened gain spectrum of the EDF, respectively.
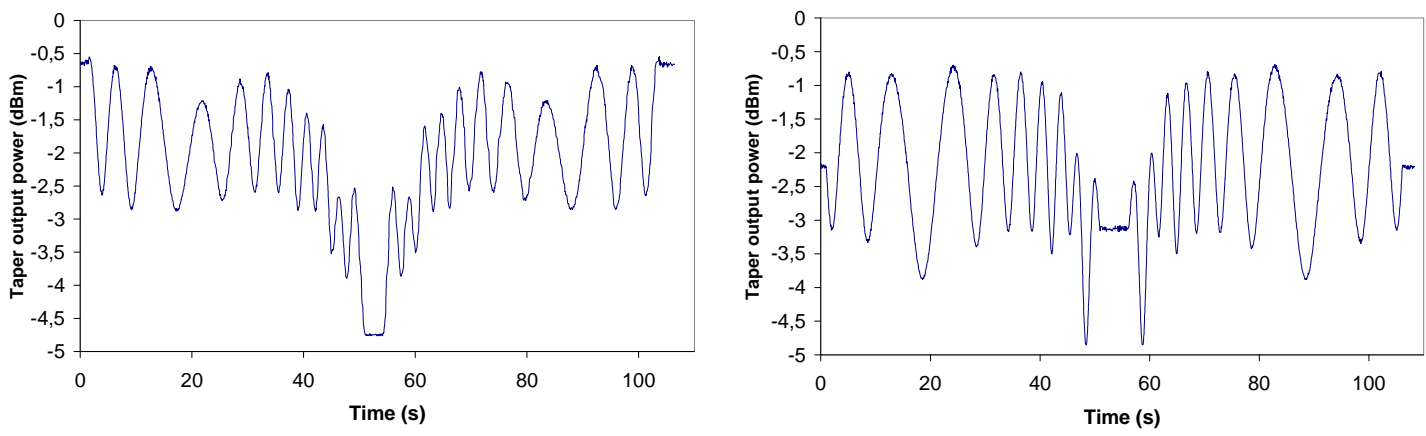

Fig. 2. Output power of the fiber taper during the displacement $(0$ to $5 \mathrm{~mm})$ and $(5$ to $0 \mathrm{~mm})$ with a constant speed for signal wavelength of $1450 \mathrm{~nm}$ and $1590 \mathrm{~nm}$. 


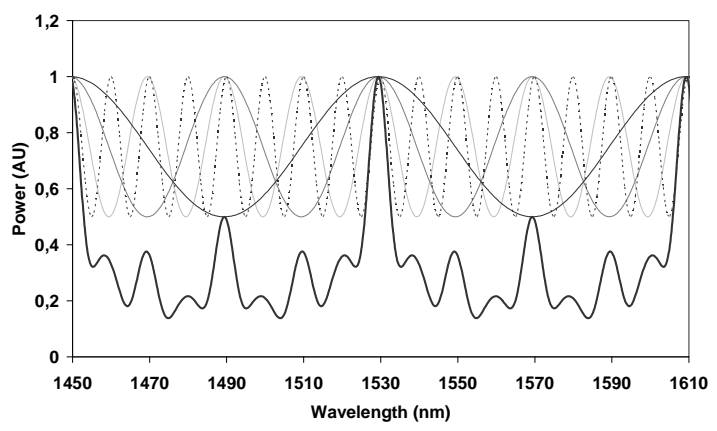

Fig. 3. Theoretical response of four filters in series as a function of the wavelength.

Each non-adiabatic fiber taper presents different response to external perturbations. As a consequence, it is very important to experimentally and individually characterize the dependence of its spectral shift on perturbations. We fabricated four different fiber tapers from the procedure described before with spectral response periods of 80, 40, 20 and $10 \mathrm{~nm}$. The shift of the spectral response of each taper as a function of the rapprochement of the taper ends has measured. The experimental results are shown in Fig. 4. In these graphics, for each value of the rapprochement of the ends of the each fiber taper, the wavelengths where the transmission of each taper is maximum are represented. Consequently, to move the wavelength of maximum transmission of the optical filter composed of the concatenated fiber tapers, a different rapprochement have to be applied to each taper.
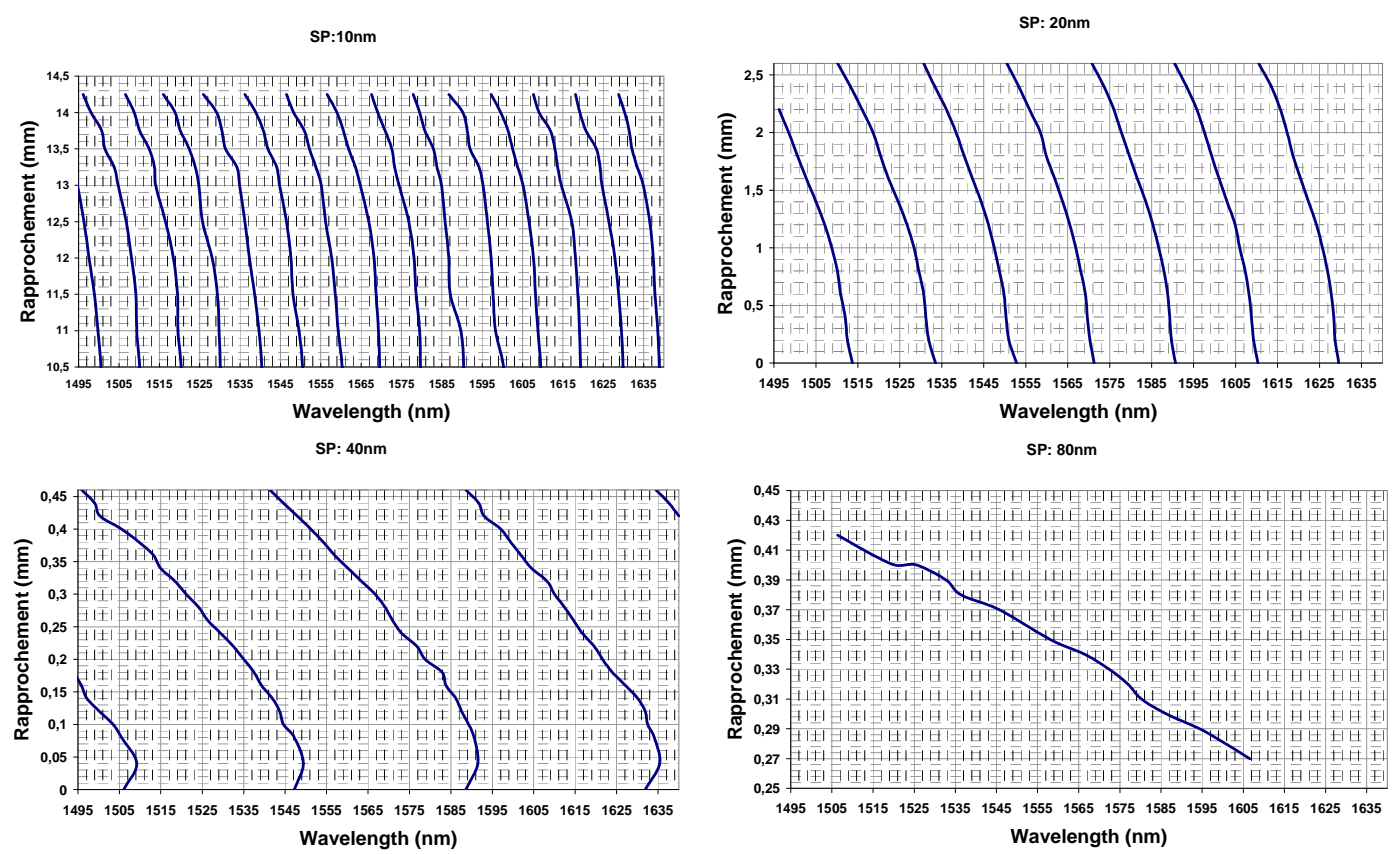

Fig. 4. Wavelengths where the transmission of each taper is maximum as a function of the rapprochement of the ends of the each fiber taper for four tapers with different spectral period (SP).

\section{EXPERIMENTAL RING FIBER LASER DEMOSTRATION}

The schematic setup of the erbium doped fiber ring laser (EDFRL) is shown in Fig. 5(a). The cavity was composed of the tunable fiber filter described before, an optical isolator, a polarization controller (PC), a 3dB optical coupler and one 1480/1550 nm WDM. The tunable filter determines the lasing wavelength of the EDFRL. An optical isolator ensures unidirectional oscillation around the ring and a polarization controller provides an intracavity mechanism to compensate for the slight polarization dependent gain. The light is coupled out through one port of the optical coupler. 

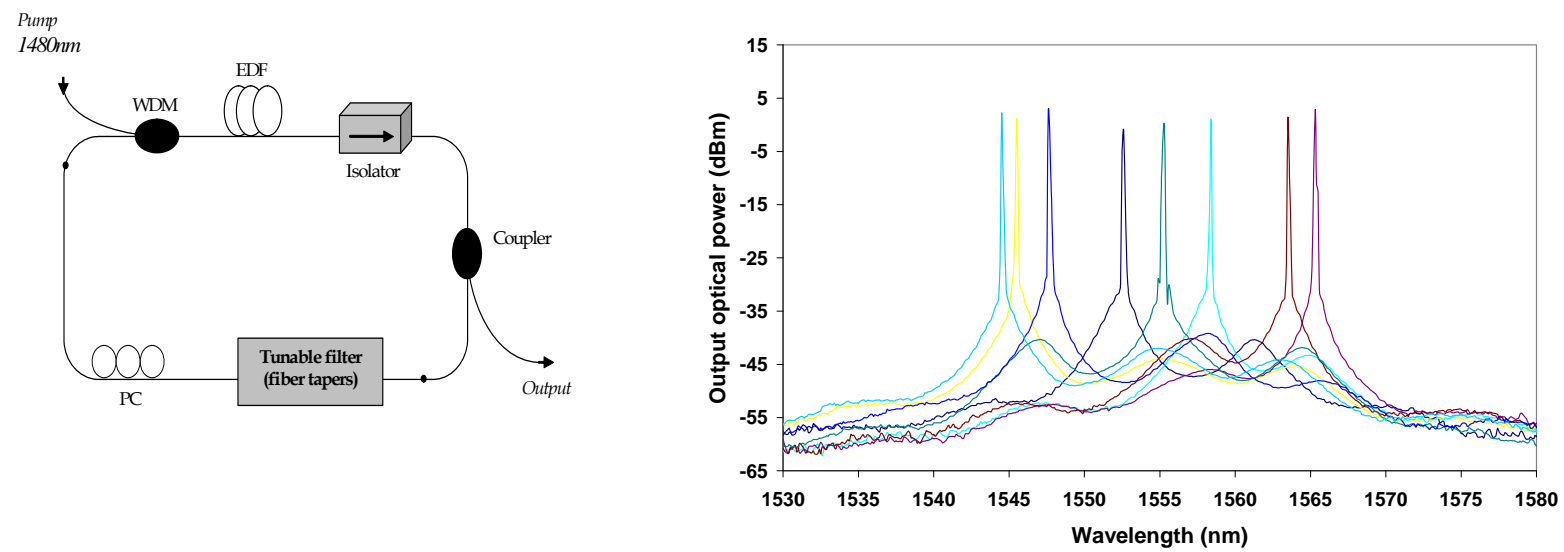

Fig. 5. (a) Experimental setups of the tunable EDFRLs: with a forward unidirectional pump configuration. (b) Output optical spectrum of the EDFRL operating in different wavelength for a total pump power of $150 \mathrm{~mW}$ at $1480 \mathrm{~nm}$.

The EDF was pumped with a $1480 \mathrm{~nm}$ pump laser. The length and peak absorption at 1530nm of the used EDF are 26m and $4.4 \mathrm{~dB} / \mathrm{m}$, respectively.

As a sample of the obtained experimental results, the output optical spectrum of the EDFRL lasing at 8 different wavelengths for a total pump power of $150 \mathrm{~mW}$ at $1480 \mathrm{~nm}$ is shown in Fig. 5(b). To achieve a specific lasing wavelength, the ends of the each fiber taper have to be moved according to the experimental results shown in Fig. 4. Moreover, as was expected, each taper have an influence in the output signal. The fiber taper with smaller spectral period (in this case 10nm) permits the fine tuning of the laser. The fiber tapers with intermediate spectral periods (in this case $20 \mathrm{~nm}$ and $40 \mathrm{~nm}$ ) reduce the second lobules. Finally, the taper with the bigger spectral period permits the optimization of the output power. The measured tuning range of the EDFRL was 20.8nm (1544.5nm-1565.3nm).

\section{CONCLUSION}

The application of the fiber taper as tunable filter in fiber lasers is presented. The spectral responses of the fiber tapers, which are quasi-sinusoidal, shift when a controlled perturbation is applied. In turn, these spectral movements depend on the spectral period of the non-adiabatic fiber tapers. The evolutions of the peaks transmission wavelengths in function of the rapprochement of the ends of the each fiber taper, for several tapers with different spectral periods, have been experimentally obtained. On the other hand, in order to obtain a narrower band-pass and a higher modulation depth of the optical filter several tapers can arranged in series. These properties were used to design an appropriate optical filter for fiber lasers. We constructed an EDFRL with four concatenated non-adiabatic fiber tapers as optical filter. The fiber laser presents a maximum tuning range of $20.8 \mathrm{~nm}$. The tuning range can be extending to the gain bandwidth of the erbium doped fiber by using techniques of the gain flattening.

\section{ACKNOWLEDGMENT}

This work was supported in part by the Spanish Government TEC2004-05936-C02, TEC2005-08218-C02-02 and TEC2007-67987-CO2-01 projects

\section{REFERENCES}

1. R. G. Lamont, D. C. Johnson and K. Hill, "Power transfer in fused biconical-taper single-mode fiber couplers: dependence on external refractive index,” Applied Optics, vol. 24, p. 6, 1985.

2. L. C. Bobb, H. D. Krumboltz and P. M. Shankar, "Pressure sensor that uses bent biconically tapered single-mode fibers," Optics Letters, vol. 16, p. 3, 1991.

3. K. Q. Kieu, M. Mansuripur, “Biconical fiber taper sensors,” IEEE Photonics Technology Letters, vol. 18, no. 21, p. $2239,2006$.

4. J. M. Corres, F. J. Arregui and I. R. Matias, “Design of humidity sensors based on tapered optical fibers,” Journal of Lightwave Technology, vol. 24, p. 8, 2006.

5. O. Frazao, R. Falate, J. L. Fabris and J. L. Santos, “Optical inclinometer based on a single long-period fiber grating combined with a fused taper,” Optics Latter, vol. 31, p. 3, 2006.

6. J. D. Love, W. H. Henry, W. J. Stewart, R. J. Black, S. Lacroix and F. Gonthier, “Tapered single-mode fibres and devices. Part1: Adiabatic Criteria,” IEEE Proceedings, vol. 138, p. 12, 1991.

7. S. Lacroix, F. Gonthier, J. Bures, “All-fiber wavelength filter from successive biconical tapers,” Optics Letters, vol. 11, no. 10, p. 671, 1986. 